\title{
Thin Film Porosity Determined by X-Rays at SEM
}

\author{
E. Ortel ${ }^{1}$, R. Kraehnert ${ }^{2}$, F. Galbert ${ }^{2}$ and V.-D. Hodoroaba ${ }^{1}$ \\ ${ }^{1}$ BAM Federal Institute for Materials Research and Testing, Berlin, Germany \\ 2 Technical University of Berlin, Berlin, Germany
}

Various applications such as automotive catalysts, photocatalysis and sensors rely on thin coatings with high active surface area. The development of new and improved coatings requires in-depth understanding of the film morphology and texture. Especially the film porosity is a key parameter to identify structure-property relationships in applications. However, an accurate porosity determination of thin porous coatings is a challenging. In this contribution we present and discuss a new approach for determining the porosity of films by x-rays at SEM.

The mass coverage of layers can be calculated by the film analysis software STRATAGem [1] from EDX determined $k$-values (the ratio of normalized intensity between unknown sample and standard) based on the well known Pouchou and Pichoir approach.[2] The high accuracy of STRATAGem results has been proven for various layered materials.[3-5] We present an approach for determination of the porosity by a combined SEM/EDX/STRATAGem analysis. From the STRATAGem-derived mass coverage and the coating thickness as determined by cross-section SEM micrographs, the average density of the film can be obtained. In a second step, the porosity can be calculated from the measured porous film density and the literature value for the pore walls bulk density. The complete approach is sketched in Figure 1.

The general procedure to determine the porosity is demonstrated on thin mesoporous $\mathrm{TiO}_{2}$ films on $\mathrm{Si}$ wafer substrates. The $\mathrm{TiO}_{2}$ films were synthesized by a template-assisted synthesis route, which provides a scalable model system with tunable porosity, see Figure 2.[6] EDX spectra of these films were measured at beam voltages of $7,10,15,20$, and $30 \mathrm{kV}$ by using a high-throughput SDD EDS detector which enables a time-efficient analysis. Polished bulk of pure Ti and Si served as standard references. Oxygen was quantified by stoichiometry as well as by using a pure $\mathrm{SiO}_{2}$ reference. The resulting $k$-values were verified by WDX analysis. The calculated mass coverage was independently validated by weighing and by ICP-OES of dissolved coatings. To determine the film thickness, the $\mathrm{TiO}_{2}$ films were cleaved and the resulting cross-section was imaged with a SEM.

The measured $k$-values for Ti K $\alpha$ and $\mathrm{Si} K \alpha$ in dependence on the beam voltage and the corresponding fitted curves calculated by STRATAGem are shown in Figure 3a for a porous $180 \mathrm{~nm}$ thick $\mathrm{TiO}_{2}$ film. Figure $3 \mathrm{~b}$ visualizes the resulting mass coverage values for all beam voltages. The scattering of the individual mass coverage values at different voltages reflects the robustness of the model.

An excellent agreement was obtained between the mass coverage calculated by STRATAGem and the mass coverage determined by weighing and ICP-OES of the same film. The increasing porosity of the thin $\mathrm{TiO}_{2}$ films illustrated in Figure 2 could be calculated quantitatively by the new approach. The validation and traceability of the porosity results will be discussed. These combined SEM/EDX/STRATAGem analyses demonstrate a unique tool for non-destructive examination of the porosity of thin porous films. 
References:

[1] Stratagem version 2.6, SAMx, 4, rue Galilée, 78280 Guyancourt, France

[2] J.-L. Pouchou, Mikrochim Acta 138 (2002), p. 133.

[3] V.-D. Hodoroaba et al., Surf. Interface Anal. 44 (2012), p. 1459.

[4] M. Procop et al., Anal. Bioanal. Chem. 374 (2002) p. 631.

[5] F. Galbert, Microsc. Microanal. 13 Suppl. 3 (2007) 96.

[6] E. Ortel et al., Small 8 (2012), p. 298.

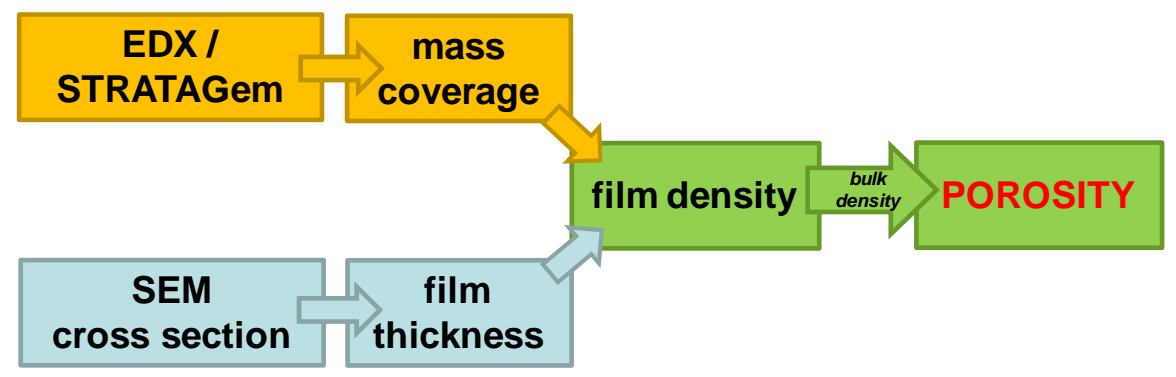

Figure 1. Illustration of the approach for the determination of the porosity of thin films by EDX/SEM analysis.
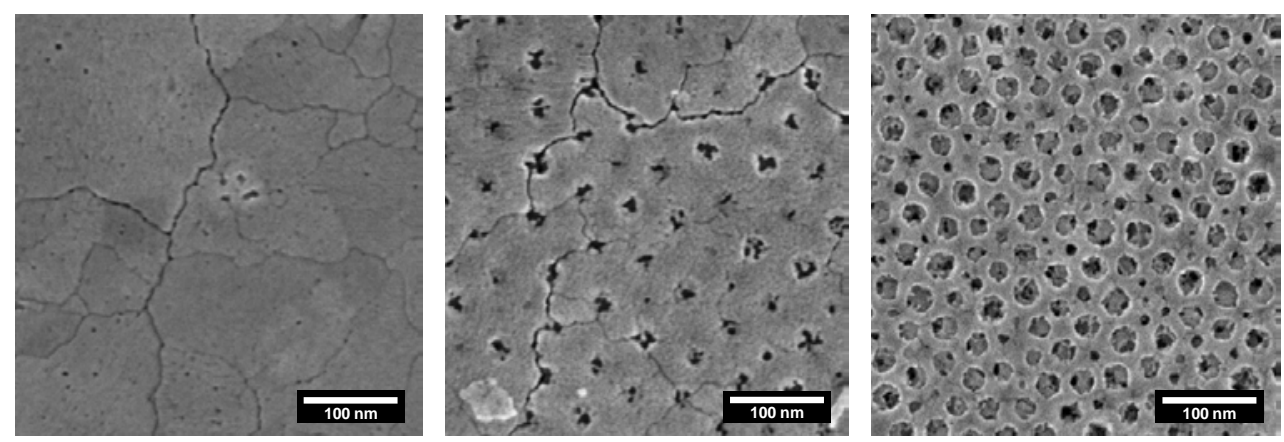

Figure 2. Top-view SEM micrographs of $\mathrm{TiO}_{2}$ films synthesized with increased amount of a mesopore template, which results in an increase of the void fraction, i.e. film porosity (from left to right).
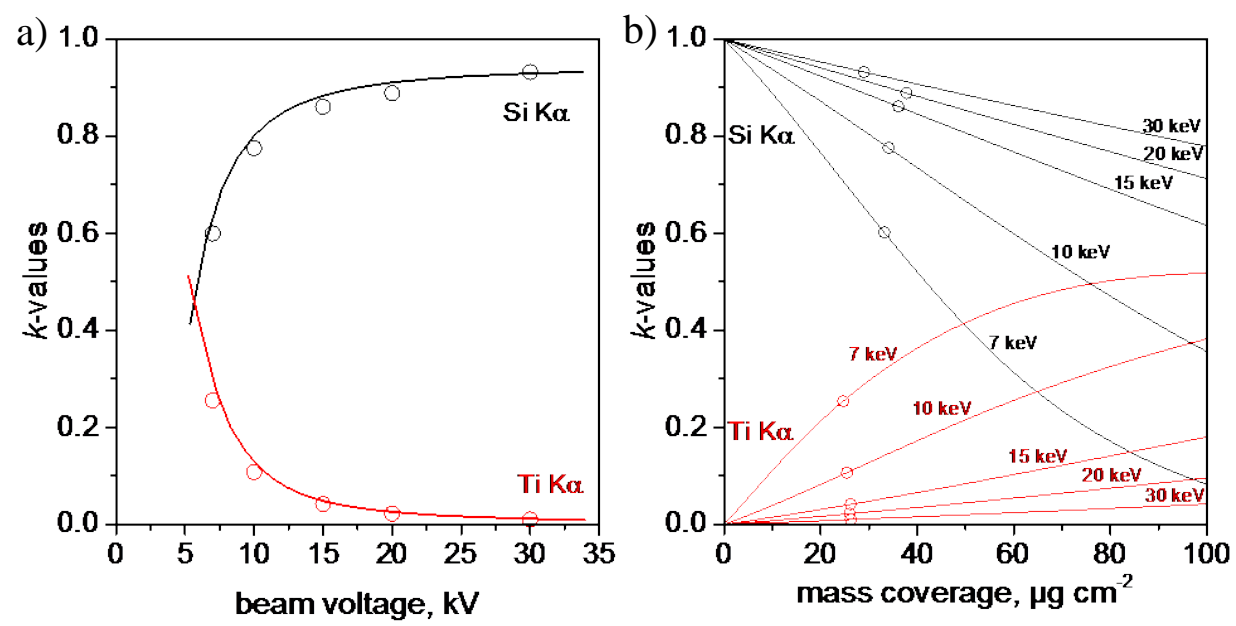

Figure 3. a) $k$-values versus beam voltage for $\mathrm{Ti} \mathrm{K} \alpha$ (red) and $\mathrm{Si} \mathrm{K} \alpha$ (black); the markers represent the

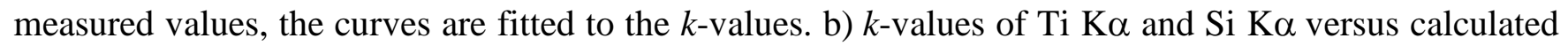
mass coverage at each beam voltage. 